\title{
Influência de fatores socioeconômicos na qualidade de vida de idosos hipertensos
}

\author{
Influence of socioeconomic factors \\ on the quality of life of elderly hypertensive individuals
}

\author{
João Marcus Oliveira Andrade ${ }^{1}$ \\ Lorena Roseli Rios ${ }^{1}$ \\ Larissa Silva Teixeira $^{1}$ \\ Fernanda Silva Vieira ${ }^{1}$ \\ Danilo Cangussu Mendes ${ }^{1}$ \\ Maria Aparecida Vieira ${ }^{1}$ \\ Marise Fagundes Silveira ${ }^{1}$
}

${ }^{1}$ Hospital Universitário Clemente de Faria, Laboratório de Pesquisa em Saúde, Universidade Estadual de Montes Claros.

Av. Cula Mangabeira 562, Santo Expedito. 39.401-001 Montes Claros MG Brasil. joao_marcus13@

hotmail.com
Abstract This study sought to evaluate the association between socioeconomic variables and the quality of life of elderly hypertensive patients treated under the Family Health Program in the city of Montes Claros, Minas Gerais, Brazil. An analytical cross study was conducted in a representative sample of 294 elderly hypertensive patients. Data were collected using a questionnaire on socioeconomic characteristics and quality of life (MINICHAL). The data were analyzed using the nonparametric Mann-Whitney and Kuskall-Wallis tests. The results showed that marital status, religion and education affect the quality of life of elderly hypertensive patients in a statistically significant way. Elderly hypertensive patients who were single/divorced/widowed, evangelical, spiritualist and belonging to other religious bodies, illiterate achieved lower scores in terms of quality of life. For the remaining variables, there was no statistical association. The conclusion, drawn is that socioeconomic factors such as marital status, education and religion influence the quality of life of elderly hypertensive patients.

Key words Elderly individuals Quality of life Hypertension, Family health, Primary health care
Resumo O presente estudo teve como objetivo analisar a associação entre fatores socioeconômicos e qualidade de vida de idosos hipertensos atendidos pelo Programa Saúde da Família na cidade de Montes Claros, Minas Gerais, Brasil. Consistiu em um estudo transversal analítico conduzido em amostra representativa de 294 idosos hipertensos. Os dados foram coletados por meio de questionário de caracterização socioeconômica e de qualidade de vida (Minichal). Foram analisados por meio de testes não paramétricos de Mann-Witney e Kuskal1-Wallis. Os resultados, por sua vez, mostraram que o estado conjugal, a religião e a escolaridade afetam de maneira estatisticamente significativa a qualidade de vida de idosos hipertensos. Idosos hipertensos solteiros/divorciados/viúvos, evangélicos, espiritas e pertencentes a outras entidades religiosas, e analfabetos apresentaram menores escores de qualidade de vida. Para as demais variáveis analisadas, não houve associação estatística. Conclui-se que os fatores socioeconômicos como estado conjugal, escolaridade e religião influenciam na qualidade de vida de idosos hipertensos.

Palavras-chave Idoso, Qualidade de vida, $\mathrm{Hi}$ pertensão, Saúde da Família, Atenção Primária à Saúde 


\section{Introdução}

A Hipertensão Arterial Sistêmica (HAS) é uma condição crônica, de origem multifatorial, que apresenta elevada prevalência na população brasileira, constituindo-se como sério fator de risco para o desenvolvimento de complicações cerebrovasculares e cardíacas. É considerada um grave problema de saúde pública em todas as regiões do mundo e no Brasil, estimando-se que tenha prevalência entre 50 e $70 \%$ entre idosos, o que a torna um dos principais determinantes de mortalidade dessa população, exigindo, assim, correta identificação do problema e a apropriada abordagem terapêutica ${ }^{1-4}$.

Inúmeros estudos avaliam o impacto da HAS na Qualidade de Vida (QV), sugerindo que o próprio quadro crônico da HAS, a terapia medicamentosa e as possíveis complicações clínicas interferem na capacidade física, emocional, interação social, atividade intelectual e atividades cotidianas, que são determinantes para o comprometimento da Qualidade de Vida ${ }^{5-8}$.

Qualidade de Vida é um conceito que vem adquirindo importância na sociedade em geral e é um constructo muito apreciado, sobretudo em relação aos adultos e idosos, considerado referencial no âmbito da saúde e no planejamento de políticas sociossanitárias para a população idosa ${ }^{9,10}$. Segundo a Organização Mundial da Saúde ${ }^{11}$, QV corresponde à "percepção do indivíduo de sua posição na vida, no contexto da sua cultura e sistema de valores em que vive, e em relação aos seus objetivos, expectativas, padrões e preocupações”.

Estudos têm evidenciado que a Qualidade de Vida de idosos hipertensos está diretamente associada a fatores socioeconômicos, como idade, gênero, nível educacional, renda, emprego e religião $0^{6-8,12,13}$. A compreensão da relação entre esses elementos mostra-se útil para subsidiar ações de saúde e condutas clínicas minimizadoras do impacto da HAS na vida de idosos.

Nesse contexto, o presente estudo teve como objetivo avaliar o impacto das condições socioeconômicas na Qualidade de Vida de idosos hipertensos atendidos pelo Programa Saúde da Família no município de Montes Claros, Minas Gerais, Brasil.

\section{Métodos}

Trata-se de estudo transversal analítico realizado no Polo de Saúde da Família Delfino Magalhães, na cidade de Montes Claros, Minas Gerais, Brasil.

O pólo Delfino Magalhães situa-se na região Oeste de Montes Claros, compreende sete uni- dades de Saúde da Família e possui cobertura de aproximadamente 30 mil habitantes, conforme dados disponibilizados pela Secretária de Saúde do município. A opção por este pólo justificouse por agrupar o maior número de unidades de Saúde da Família em relação aos demais pólos; possuir o maior número de idosos hipertensos cadastrados; e por acessibilidade geográfica para os pesquisadores.

Participaram do estudo 294 idosos com idade igual ou superior a 60 anos completos ${ }^{14}$, com diagnóstico médico comprovado há pelo menos um ano para Hipertensão Arterial Sistêmica, e residentes em áreas de atuação de unidades do Programa Saúde da Família.

A seleção da amostra do estudo constituiu-se em duas etapas. Em um primeiro momento, realizou-se cadastro prévio de todos os indivíduos com idade igual ou superior a 60 anos e hipertensos ${ }^{14}$ há pelo menos um ano, mediante prontuário médico. Esse cadastro foi realizado com auxílio de Agentes Comunitários de Saúde, previamente orientados. Por meio desse cadastro obteve-se a relação de todos os idosos hipertensos residentes no polo, totalizando 1291 indivíduos. Nesse momento, em virtude das características dos questionários usados neste estudo, foram excluídos aqueles indivíduos sabidamente acamados, inconscientes, institucionalizados e sem condições gerais para responder aos questionários.

O cálculo amostral para população finita foi realizado considerando-se os seguintes dados: a) número total de idosos hipertensos, conforme levantamento prévio $(\mathrm{n}=1291)$; b) prevalência estimada do evento em $45 \%{ }^{2}$; c) intervalo de confiança de 95\%; d) erro fixado de 5\%. Os cálculos evidenciaram um tamanho mínimo amostral (n) de 294 participantes, considerados sujeitos deste estudo. A seleção desses sujeitos da pesquisa foi realizada de forma aleatória simples através do software Statistical Package for the Social Sciences - SPSS for Windows, versão 18.0. Aqueles indivíduos que não concordaram em participar da pesquisa, não responderam o questionário em sua plenitude ou não foram encontrados no domicílio após 3 tentativas, foram substituídos também de forma aleatória, segundo a aplicação do software supracitado.

Os instrumentos de coleta de dados foram aplicados por equipe composta por oito entrevistadores voluntários, após processo de calibração realizado pelos coordenadores da pesquisa e mediante visitas domiciliares realizadas aos sujeitos. Foi realizado previamente, estudo piloto em população pertencente a outro polo de Saúde da Família da cidade de Montes Claros, para viabilização de 
estratégias de aplicação dos questionários aos sujeitos desta investigação. Os dados foram coletados entre os meses de julho a dezembro de 2012.

Para avaliação da qualidade de vida foi utilizado o Mini-Cuestionario de Calidad Vida em Hipertensión Arterial - Minichal, na versão traduzida e validada no Brasil, por ser o questionário específico para avaliação da qualidade de vida a indivíduos hipertensos, de fácil e rápida aplicação ${ }^{15}$.

O Minichal é composto por 16 itens, sendo 10 referentes ao domínio "Estado Mental” (state of mind) e seis itens ao domínio "Manifestações Somáticas" (somatic manifestations). Os itens devem ser respondidos em referência aos últimos sete dias antecedentes à aplicação do questionário. A escala de pontuação é do tipo Likert com quatro possíveis respostas ( $0=$ não, absolutamente; $1=$ sim, um pouco; $2=\operatorname{sim}$, médio; $3=\operatorname{sim}$, muito). Os pontos variam de 0 (melhor nível de saúde) a 30 (pior nível de saúde) para o domínio "Estado Mental”, e para o domínio "Manifestações Somáticas” variam de 0 (melhor nível de saúde) a 18 (pior nível de saúde $)^{15}$. No entanto, neste trabalho, o escore original do instrumento foi convertido para uma escala de zero a 100, sendo zero o pior nível e 100, o melhor nível de qualidade de vida relacionada à saúde. Essa conversão foi necessária para que se pudesse comparar os resultados aos questionários gerais de avaliação da qualidade de vida ${ }^{16}$.

Para caracterização do perfil socioeconômico e de saúde dos sujeitos, foi elaborado o Questionário de Caracterização de Idosos Hipertensos, tomando como subsídios estudo de Damy ${ }^{17}$, a fim de coletar informações referentes a sexo, idade, escolaridade, renda, situação conjugal, apoio familiar, condições da habitação, núcleo familiar, atividade profissional, cobertura previdenciária, prática de atividade física, morbidades autorreferidas, utilização de medicamentos, sono, tabagismo, ingestão de bebidas alcoólicas e utilização de serviços de saúde.

A análise de dados foi conduzida por meio da análise bivariada e de testes não paramétricos Mann-Witney e Kuskall-Wallis, para verificar associação entre as variáveis independentes e dependentes. As medidas de qualidade de vida (escores dos domínios do Minichal) variam de 0 a 100 e foram consideradas variáveis dependentes e as características do perfil socioeconômico foram consideradas variáveis independentes. As variáveis foram descritas através de medidas de tendência central e dispersão. Para as análises foi utilizado o software SPSS. O desenvolvimento do estudo foi aprovado e atendeu às normas nacionais e internacionais de ética em pesquisa envolvendo seres humanos.

\section{Resultados}

Participaram deste estudo 294 idosos hipertensos, residentes em áreas de atuação de unidades do Programa Saúde da Família, na cidade de Montes Claros, Minas Gerais, Brasil. As características socioeconômicas dos participantes estão apresentadas na Tabela 1 e mostram que a maioria dos sujeitos era do sexo feminino $(\mathrm{n}=180 ; 61,2 \%)$, com idade entre 60-69 anos $(\mathrm{n}=147 ; 50 \%)$, casados/amasiados ( $\mathrm{n}=174 ; 59,2 \%)$, católicos ( $\mathrm{n}$ $=201 ; 68,4 \%)$, alfabetizados $(\mathrm{n}=159 ; 54,1 \%)$ e com renda familiar entre 1 e 2 salários mínimos ( $\mathrm{n}=201 ; 68,4 \%)$.

A associação entre variáveis socioeconômicas e o domínio I (Estado Mental) mostrou-se esta-

Tabela 1. Fatores socioeconômicos de idosos hipertensos, Montes Claros, Minas Gerais. Julho, 2012.

\begin{tabular}{|c|c|c|}
\hline Variável & $\mathbf{n}$ & $\%$ \\
\hline \multicolumn{3}{|l|}{ Sexo } \\
\hline Masculino & 114 & 38,8 \\
\hline Feminino & 180 & 61,2 \\
\hline Total & 294 & 100,0 \\
\hline \multicolumn{3}{|l|}{ Idade } \\
\hline 60 a 69 anos & 147 & 50 \\
\hline 70 a 79 anos & 102 & 34,7 \\
\hline$>80$ anos & 45 & 15,3 \\
\hline Total & 294 & 100,0 \\
\hline \multicolumn{3}{|l|}{ Estado conjugal } \\
\hline Casado/Amasiado & 174 & 59,2 \\
\hline Solteiro/Divorciado/Viúvo & 120 & 40,8 \\
\hline Total & 294 & 100,0 \\
\hline \multicolumn{3}{|l|}{ Trabalho } \\
\hline Sim & 30 & 10,2 \\
\hline Não & 264 & 89,8 \\
\hline Total & 294 & 100,0 \\
\hline \multicolumn{3}{|l|}{ Religião } \\
\hline Católica & 201 & 68,4 \\
\hline Evangélica/Espírita/Outras & 93 & 31,6 \\
\hline Total & 294 & 100,0 \\
\hline \multicolumn{3}{|l|}{ Escolaridade } \\
\hline Analfabeto & 135 & 45,9 \\
\hline Alfabetizado & 159 & 54,1 \\
\hline Total & 294 & 100,0 \\
\hline \multicolumn{3}{|l|}{ Renda familiar } \\
\hline Menor que 1 SM & 33 & 11,2 \\
\hline Entre $1 \mathrm{e} 2 \mathrm{SM}$ & 201 & 68,4 \\
\hline$>3 \mathrm{SM}$ & 60 & 20,4 \\
\hline Total & 294 & 100,0 \\
\hline
\end{tabular}

Fonte: Prontuários de atendimento dos adolescentes em cumprimento de medida socioeducativa de internação na UIPP (DF) usuários de medicamento psicotrópico entre os meses de julho a outubro de 2010. 
tisticamente significativa para estado conjugal ( $\mathrm{p}$ $=0,029)$, religião $(\mathrm{p}=0,032)$ e escolaridade $(\mathrm{p}$ $=0,045)$.

A Tabela 2 mostra que idosos hipertensos solteiros/divorciados/viúvos apresentaram médias gerais de qualidade de vida menores $(85,11 ; \mathrm{DP}=$ $10,08)$, quando comparados com aqueles na condição de casados/amasiados $(87,92 ; \mathrm{DP}=8,15)$. A religião também influenciou na qualidade de vida dos sujeitos do estudo. Assim, aqueles autodeclarados como evangélicos, espíritas e pertencentes a outras entidades religiosas apresentam médias de qualidade de vida inferiores aos idosos definidos como católicos $(85,76 ; \mathrm{DP}=8,31$ vs. 87,21; $\mathrm{DP}=$ $9,3)$. Acerca da variável escolaridade, idosos analfabetos apresentaram médias inferiores de qualidade de vida $(85,45 ; \mathrm{DP}=9,87)$ quando comparados com aqueles alfabetizados $(87,91 ; \mathrm{DP}=8,19)$.

Em relação ao domínio 2 (Manifestações Somáticas), os dados analisados mostraram associação estatisticamente significativa apenas para a variável escolaridade. Os resultados mostram, que os idosos hipertensos analfabetos, apresentam menores médias de qualidade de vida, quando comparados com aqueles alfabetizados $(83,45 ; \mathrm{DP}$ $=13,17$ e 86,$67 ; \mathrm{DP}=11,37)$, respectivamente $(\mathrm{p}$ $=0,034)$. (Tabela 2)

Ao se analisar o domínio 3 (Estado Geral de Saúde), que consiste na média geral do Minichal (domínios 1 e 2), encontrou-se associação estatisticamente significativa para as variáveis estado conjugal ( $\mathrm{p}=0,049)$ e escolaridade $(\mathrm{p}=0,049)$. Por meio da Tabela 2, observa-se que, idosos hipertensos na condição de solteiros/divorciados/viúvos apresentaram médias gerais de qualidade de vida menores $(76,11 ; \mathrm{DP}=11,78)$, quando comparados com aqueles na condição de casados/amasiados $(79,15 ; \mathrm{DP}=9,47)$. Acerca da variável escolaridade, idosos analfabetos apresentaram médias inferiores $(76,38 ; \mathrm{DP}=11,43)$ quando comparados com aqueles alfabetizados $(79,24 ; \mathrm{DP}=9,60)$.

\section{Discussão}

A qualidade de vida é um conceito subjetivo que sofre influência de inúmeros fatores inerentes ao ser humano, como físico, mental e ambiental.

Tabela 2. Fatores socioeconômicos associados a escores de qualidade de vida, por domínios do MINICHAL entre idosos hipertensos, Montes Claros, Minas Gerais. Julho, 2012.

\begin{tabular}{|c|c|c|c|c|c|c|}
\hline \multirow[b]{3}{*}{ Variáveis } & \multicolumn{6}{|c|}{ Manifestações } \\
\hline & \multicolumn{2}{|c|}{ Estado Mental } & \multicolumn{2}{|c|}{ Somáticas } & \multicolumn{2}{|c|}{ Domínio Global } \\
\hline & Média (d.p) & Valor $\mathbf{p}$ & Média (d.p) & Valor $\mathbf{p}$ & Média (d.p) & Valor $\mathrm{p}$ \\
\hline Sexo & & 0,068 & & 0,655 & & 0,493 \\
\hline Masculino & $87,68(8,9)$ & & $84,5(13,22)$ & & $78,49(10,22)$ & \\
\hline Feminino & $86,2(9,16)$ & & $85,64(11,71)$ & & $77,56(10,78)$ & \\
\hline \multicolumn{7}{|l|}{ Idade } \\
\hline 60 a 69 anos & $88.01(7,97)$ & 0,173 & $86,13(11,89)$ & 0,255 & $79,16(9,80)$ & 0,096 \\
\hline 70 a 79 anos & $85,66(10,09)$ & & $84,89(12,33)$ & & $77,47(10,87)$ & \\
\hline$>80$ anos & $85,22(9,73)$ & & $82,78(13,51)$ & & $74,88(11,72)$ & \\
\hline Estado conjugal & & $0,029^{*}$ & & 0,333 & & $0,049^{*}$ \\
\hline Casado/Amasiado & $87,92(8,15)$ & & $85,88(11,73)$ & & $79,15(9,47)$ & \\
\hline Solteiro/Divorciado/Viúvo & $85,11(10,08)$ & & $84,18(13,11)$ & & $76,11(11,78)$ & \\
\hline Trabalho & & 0,142 & & 0,052 & & 0,108 \\
\hline Sim & $89,5(6,24)$ & & $88,74(11,16)$ & & $81,63(6,68)$ & \\
\hline Não & $86,41(9,31)$ & & $84,78(12,39)$ & & $77,5(10,84)$ & \\
\hline Religião & & $0,032^{\star}$ & & 0,318 & & 0,483 \\
\hline Católico & $87,21(9,38)$ & & $85,55(12,46)$ & & $78,04(10,83)$ & \\
\hline Evangélica/Espírita/Outras & $85,76(8,31)$ & & $84,3(12,19)$ & & $77,59(10,18)$ & \\
\hline Escolaridade & & $0,045^{*}$ & & $0,034^{*}$ & & $0,049^{*}$ \\
\hline Analfabeto & $85,45(9,87)$ & & $83,45(13,17)$ & & $76,38(11,43)$ & \\
\hline Alfabetizado & $87,91(8,19)$ & & $86,67(11,37)$ & & $79,24(9,60)$ & \\
\hline \multicolumn{7}{|l|}{ Renda familiar } \\
\hline Menor que $1 \mathrm{SM}$ & $86,29(9,06)$ & 0,663 & $85,59(9,15)$ & 0,661 & $76,73(10,58)$ & 0,275 \\
\hline Entre 1 e 2 SM & $86,66(9,36)$ & & $84,8(12,67)$ & & $77,61(10,40)$ & \\
\hline$>3 \mathrm{SM}$ & $87,92(8,25)$ & & $85,84(13,10)$ & & $79,43(11,55)$ & \\
\hline
\end{tabular}


$\mathrm{O}$ conceito de qualidade de vida relacionado à saúde procura limitar o estudo desses fatores para aqueles mais diretamente ligados à condição física, psíquica e social do indivíduo. A avaliação da qualidade de vida de indivíduos idosos portadores de Hipertensão Arterial Sistêmica, a partir do Minichal, corresponde a uma tentativa de medir os principais fatores ligados à HAS, que podem influenciar a sensação de bem-estar do indivíduo. Essa abordagem é importante, pois pode orientar as intervenções em saúde para aspectos que possam impactar positivamente a qualidade de vida ${ }^{15}$.

Os resultados deste estudo mostraram influência das condições socioeconômicas na qualidade de vida dos idosos hipertensos. Em todos os domínios, pelo menos uma variável esteve associada a menores escores de qualidade de vida. No entanto, os resultados revelaram que o estado conjugal, religião e a escolaridade afetam de maneira estatisticamente significativa a qualidade de vida de idosos hipertensos do Pólo de Saúde da Família Delfino Magalhães, em Montes Claros, Minas Gerais, Brasil.

Alguns estudos discutem a relação entre hipertensão, fatores socioeconômicos e qualidade de vida $a^{7,8,13,18,19}$. No entanto, na visão do idoso hipertenso, poucos são os registros na literatura nacional e internacional que os relacionam por meio do Minichal na Estratégia Saúde da Família.

A escolaridade constitui-se um dos principais fatores relacionados à qualidade de vida em diferentes grupos populacionais ${ }^{8,12,20}$. Sprangers et al. ${ }^{21}$ mostraram em estudo conduzido com portadores de doenças crônicas, entre elas a HAS em idosos, que o baixo nível de escolaridade esteve relacionado a baixos níveis de qualidade de vida.

A baixa escolaridade compromete o acesso à educação em saúde, estratégia que possibilita a adoção de comportamentos saudáveis e a mobilização social para a melhoria das condições de vida, influencia a adesão ao tratamento de condições crônicas, como a HAS, em virtude da relação com menores condições econômicas e acesso a serviços de saúde ${ }^{19,22}$.

A baixa escolaridade, comum às diferentes regiões do Brasil, pode representar dificuldades no entendimento das orientações realizadas pela Estratégia Saúde da Família, assim como no seguimento do tratamento, constituindo-se em importante indicador a ser observado pelos gestores, e profissionais de saúde no planejamento de estratégias, programas, propostas e ações destinadas a este grupo populacional ${ }^{23}$.

Estudo desenvolvido por Lasheras et al. ${ }^{24}$, que buscou analisar o impacto da escolaridade na qualidade de vida, mostrou forte associação entre baixos níveis educacionais e infelicidade, fracas relações sociais, baixa percepção de autocuidado e saúde, e problemas sensoriais entre os idosos. A educação é um importante indicador associado a auto nível social e econômico, que têm impactos diretos na qualidade de vida de idosos ${ }^{25}$.

Considerando o estado civil, os indivíduos idosos com cônjuges apresentaram melhores escores de qualidade de vida e estado de saúde do que aqueles sem cônjuge ${ }^{26}$. Idosos casados ou em união consensual apresentaram maior pontuação nos domínios "capacidade funcional”, "aspectos físicos", "estado geral de saúde", "vitalidade”, "aspectos sociais" e "saúde mental" por meio do questionário genérico de qualidade de vida SF-36. Assim, apresentaram melhor qualidade de vida quando comparados aos separados ou viúvos ${ }^{26}$.

No presente estudo, houve associação entre o domínio estado mental e para a média geral do Minichal. Estudo conduzido por Perkins et al. ${ }^{22}$ apresentou melhores escores de qualidade de vida para idosos vivendo com cônjuge em relação àqueles sozinhos. No entanto, outros estudos não encontraram influência do estado civil na qualidade de vida de idosos ${ }^{8,12,19}$.

A baixa participação social e o estado de viver sozinho é freqüentemente associado a baixa qualidade de vida ${ }^{27,28}$. Problemas de saúde psicossocial e sentimentos de solidão são mais comuns entre aqueles que vivem sozinhos, devido à falta de apoio emocional dentro da família e na sociedade ${ }^{1}$.

No entanto, outros estudos apresentam diferentes perspectivas e afirmam que o viver sozinho não é necessariamente o mesmo que sentir solidão e parece não estar associado a menores índices de qualidade de vida ${ }^{1,27}$. Acredita-se que baixos níveis de saúde podem ser motivos que o idoso passe a viver com familiares ou em instituições asilares. Esse novo estado pode comprometer o status de saúde e diminuir os níveis de qualidade de vida ${ }^{29}$.

Estudo indicou que idosos pertencentes a religiões evangélicas obtiveram menores escores de qualidade de vida do que aqueles declarados como católicos $^{12}$. No entanto, os fatores atribuíveis ainda não são claros. É possível que os indivíduos com pior estado de saúde migram de uma religião para outra em busca de maior apoio espiritual ${ }^{12}$. Zaitune et al. $^{2}$ ao estudarem a relação entre filiação religiosa e estado de saúde, não encontraram associação com a prevalência de hipertensão arterial entre idosos. $\mathrm{O}$ maior envolvimento religioso está associado com melhor saúde mental, podendo impactar positivamente nos escores de qualidade de vida ${ }^{12}$.

No presente estudo, a análise da associação entre fatores socioeconômicos e qualidade de vida 
não revelou associação estatisticamente significativa para sexo, idade, trabalho e renda. A análise da qualidade de vida relacionada ao sexo mostrou que as mulheres possuem menores escores de qualidade de vida em relação aos homens em quase todos os domínios do SF-36 $6^{8,9,12}$.

Em uma amostra de 1.688 indivíduos na China, Li et al. ${ }^{19}$ encontraram escores mais baixos entre as mulheres na seguintes dimensões: funcionamento físico, dor, estado geral de saúde e vitalidade. No Brasil, os estudos publicados sobre a auto-avaliação da saúde com uma pergunta geral encontrou uma pior auto-avaliação da saúde entre as mulheres quando comparadas a homens $s^{30-32}$.

É importante destacar que neste estudo a idade não foi fator de risco significativo para baixos escores de qualidade de vida, embora a literatura aponte que com o aumento da idade haja uma redução dos escores de qualidade de vida ${ }^{33-35}$.

Uma possível explicação seria que, nesta investigação, a comparação foi realizada entre indivíduos de uma mesma faixa etária, enquanto outros trabalhos realizam comparações entre indivíduos jovens e adultos com idosos. Apesar do declínio da qualidade de vida com o aumento da idade, nem todos os idosos a relatam baixa; como envelhecimento, há um declínio nos aspectos físicos da qualidade de vida, porém pode não haver mudanças, ou apenas discretas alterações, nos aspectos mentais, podendo assim serem explicadas as diferenças no número de pessoas idosas que experimentam uma qualidade de vida boa ou aceitável ${ }^{34}$.

Além disso, a renda não esteve significativamente associada aos escores de qualidade de vida neste estudo, embora a literatura considere esses fatores como influentes ${ }^{35}$. De acordo com Jakobsson et al. ${ }^{34}$ a renda se reduz acentuadamente quando ocorre a aposentadoria, e os fatores socioeconômicos podem influenciar a qualidade de vida, tendo sido encontradas associações entre melhores condições socioeconômicas e melhores escores de qualidade de vida.

\section{Conclusão}

O presente estudo revelou que fatores socioeconômicos como estado conjugal, escolaridade e religião influenciam na qualidade de vida de idosos hipertensos. Reconhecendo que a hipertensão arterial é uma condição clínica irreversível e com grandes impactos sobre a vida do idoso, a adoção de ações e estratégias voltadas para a minimização e/ou reversão dos efeitos negativos atrelados a fatores socioeconômicos pode tornar-se uma forte estratégia de melhoria de indicadores de qualidade de saúde e de vida de idosos.

\section{Colaboradores}

JMO Andrade, LR Rios, LS Teixeira, FS Vieira, DC Mendes, MA Vieira e MF Silveira participaram igualmente de todas as etapas de elaboração do artigo. 


\section{Agradecimentos}

Aos colaboradores responsáveis pela coleta de dados, alunos do curso de graduação em Enfermagem da Universidade Estadual de Montes Claros. A Secretaria Municipal de Saúde de Montes Claros pela disponibilização de dados. Aos Agentes Comunitários de Saúde das unidades envolvidas pela ajuda nas etapas iniciais do estudo.

\section{Referências}

1. Brito DMS, Araújo TL, Galvão MTG, Moreira TMM, Lopes MVO. Qualidade de vida e percepção da doença entre portadores de hipertensão arterial. Cad Saude Publica 2008; 24(4):933-940.

2. Zaitune MPA, Barros MBA, Cézar CLG, Carandina L, Goldbaum M. Hipertensão arterial em idosos: prevalência, fatores associados e práticas de controle no município de Campinas, São Paulo, Brasil. Cad Saude Publica 2006; 22(2):285-294.

3. Neder MM, Borges AAN. Systemic hypertension in Brazil: how much have we improved our knowledge about its epidemiology? Rev Bras Hipertens 2006; 13(2):126-133.

4. Youssef RM, Moubarak II, Kamel MI. Factors affecting the quality of life of hypertensive patients. East Mediterr Health J 2005; 11(1-2):109-118.

5. Arbex FS, Almeida EA. Qualidade de vida e hipertensão arterial no envelhecimento. Rev Bras Clin Med 2009; 7(5):339-342.

6. Carvalho ED, Valadares ALR, Costa-Paiva LH, Pedro AO, Morais SS, Pinto-Neto AM. Atividade física e qualidade de vida em mulheres com 60 anos ou mais: fatores associados. Rev. Bras. Ginecol. Obstet. 2010; 32(9):433-440.

7. Wang HM, Beyer M, Gensichen J, Gerlach FM. Health-related quality of life among general practice patients with differing chronic diseases in Germany: cross sectional survey. BMC Public Health 2008; 8:246.

8. Wang R, Zhao Y, He X, Ma X, Yan X, Sun Y, Liu W, Gu Z, Zhao J, He J. Impact of hypertension on health-related quality of life in a population-based study in Shanghai, China. Public Health 2009; 123(8):534-539.

9. Castellón A, Pino S. Calidad de vida en la atención al mayor. Revista Multidisciplinar de Gerontología 2003; 13(3):188-192.

10. Campolina AG, Pinnheiro MM, Ciconelli RM, Ferraz MB. Quality of life among the Brazilian adult population using the generic SF-8 questionnaire. Cad Saude Publica 2011; 27(6):1121-1131.

11. The World Health Organization. Quality of Life assessment (WHOQOL): position paper from the World Health Organization. Soc Sci Med 1995; 41(10):14031409.

12. Lima MG, Barros MBA, César CLG, Goldbaum M, Carandina L, Ciconelli RM. Health related quality of life among the elderly: a population-based study using SF-36 survey. Cad Saude Publica 2009; 25(10):2159-2167.

13. Lam CL, Lauder IJ. The impact of chronic diseases on the health-related quality of life (HRQOL) of Chinese patients in primary care. Fam Pract 2000; 17(2):159-166.

14. Organización Mundial De La Salud (OMS). Aplicacion de La epidemiologia al estúdio de los ancianos: informe de um grupo científico de la OMS. Genebra: OMS; 1984. (Série Informes Técnicos, n. 706).

15. Schulz RB, Rossignoli P, Correr CJ, Fernández-Llimós F, Detoni PM. Validation of the short form of spanish hypertension quality of life questionnaire (Minichal) for portuguese (Brasil). Arq Bras Cardiol. 2007; 90(2):139144.

16. Merchiors AC, Correr CJ, Pontarolo R, Santos FOS, Souza RAP. Qualidade de Vida em Pacientes Hipertensos e Validade Concorrente do Minichal-Brasil. Arq Bras Cardiol 2010; 94(3):357-364.

17. Damy AJC. Perfil multidimensional e avaliação da capacidade funcional em idosos de baixa renda [tese]. São Paulo: Universidade de São Paulo; 2010. 
18. Bardage C, Isacson DG. Hypertension and health-related quality of life: an epidemiological study in Sweden. JClin Epidemiol 2001; 54(2):172-181.

19. Li W, Liu L, Puente JG, Li Y, Jiang X, Jin S, Ma H, Kong L, Ma L, He X, Ma S, Chen C. Hypertension and health-related quality of life: an epidemiological study in patients attending hospital clinics in China. J Hypertens 2005; 23(9):1667-1676.

20. Roca-Cusachs A, Badia X, Dalfo A, Gascon G, Abellan J, Lahoz R, Varela C, Velasco O. Relationship between clinical and therapeutic variables and health-related quality of life in patients with hypertension. Minichal Study. Med Clin (Barc) 2003; 121(1):12-17.

21. Sprangers MA, Regt EB, Andries F, Van Agt HM, Bijl RV, Boer JB, Foets M, Hoeymans N, Jacobs AE, Kempen GI, Miedema HS, Tijhuis MA, de Haes HC. Which chronic conditions are associated with better or poorer quality of life? J Clin Epidemiol 2000; 53(9):895-907.

22. Perkins AJ, Stump TE, Monahan PO, McHorney CA. Assessment of differential item functioning for demographic comparisons in the MOS SF-36 health survey. Qual Life Res 2006; 15(3):331-348.

23. Pereira RJ, Cotta RMM, Franceschini SCC, Ribeiro RCL, Sampaio RF, Priore SE, Cecon PR. Influência de fatores sociossanitários na qualidade de vida dos idosos de um município do Sudeste do Brasil. Cien Saude Colet 2011; 16(6):2907-2917.

24. Lasheras C, Patterson AM, Casado C, Fernandez S. Effects of education on the quality of life, diet, and cardiovascular risk factors in an elderly Spanish community population. Exp Aging Res 2001; 27(3):257-270.

25. Szwarcwald CL, Souza-Júnior PRB, Esteves MAP, Damacena GN, Viacava F. Socio-demographic determinants of self-rated health in Brazil. Cad Saude Publica 2005; 21(Supl.):S54-S64.

26. Vahdaninia M, Goshtasebi A, Montazeri A, Maftoon F. Health-related quality of life in an elderly population in Iran: a population-based study. Payesh 2005; 4:113-120.
27. Victor C, Scambler S, Bond J, Bowling A. Loneliness in later life. In: Walker A, Hagen Hennessy C, editors. Growing Older: Quality of Life in Older Age. Maidenhead: Open University Press; 2004.

28. Walker A, Hagen Hennessy C, editors. Growing Older: Quality of Life in Older Age. Maidenhead: Open University Press; 2005.

29. Gallicchio L, Hoffman SC, Helzlsouer KJ. The relationship between gender, social support, and health-related quality of life in a community-based study in Washington County Maryland. Qual Life Res. 2007; 16(5):777-786.

30. Lima MG, Barros AMB, César CLG, Goldbaum M, Carandina L, Alves MCGP. Health-related behavior and quality of life among the elderly: a population-based study. Rev Saude Publica 2011; 45(3):485-493.

31. Barros MBA, César CLG, Carandina L, Torre GD. Desigualdades sociais na prevalência de doenças crônicas no Brasil, PNAD-2003. Cien Saude Colet 2006; 11(4):911-26.

32. Pinheiro RS, Viacava F, Travassos C, Brito AS. Gênero, morbidade, acesso e utilização de serviços de saúde no Brasil. Cien Saude Colet 2002; 7(4):687-707.

33. Michelson H, Bolund C, Brandberg Y. Multiple chronic health problems are negatively associated with health-related quality of life (HRQoL) irrespective of age. Qual Life Reses 2001; 9(10):1093-1104.

34. Jakobsson U, Hallberg IR, Westergren A. Overall and health related quality of life among the oldest old in pain. Qual Life Reses 2004; 1(13):125-136.

35. García LE, Banegas B, Pérez-Regadera G, Cabrera H, Rodríguez-Artalejo F. Social network and health related quality of life in older adults: a population-based study in Spain. Qual Life Reses 2005; 14(2):4-40.

Artigo apresentado em 17/10/2013

Aprovado em 28/03/2014

Versão final apresentada em 05/04/2014 